\title{
Internal representation and memory formation of odor preference based on oscillatory activities in a terrestrial slug
}

\author{
Tatsuhiko Sekiguchi, ${ }^{1,4}$ Hiroyuki Furudate, ${ }^{2}$ and Tetsuya Kimura ${ }^{3}$ \\ ${ }^{1}$ Honda Research Institute Japan, Saitama 351-0188, Japan; ${ }^{2}$ Graduate School of Science and Engineering, Saitama University, \\ Saitama 338-8570, Japan; ${ }^{3}$ Laboratory for Alzheimer's Disease, Brain Science Institute, RIKEN, Saitama 351-0198, Japan
}

\begin{abstract}
The terrestrial slug Limax exhibits a highly developed ability to learn odors with a small nervous system. When a fluorescent dye, Lucifer Yellow (LY), is injected into the slug's body cavity after odor-taste associative conditioning, a group of neurons in the procerebral (PC) lobe, an olfactory center of the slug, is labeled by LY. We examined the relationships between conditioning strategies and LY labeling. The positions of LY-labeled neurons in the PC lobe after appetitive conditioning were more apical than those after aversive conditioning and did not depend on the conditioned odor, suggesting that the biological value of odors affected the position of LY-labeled neural clusters. A simple computational model consisting of two layers of oscillators with electrical synaptic interaction was constructed based on physiological features of the PC lobe, and showed that the oscillatory phase difference between the layers contributed to determination of the positions of LY-labeled neurons, suggesting that phase difference in oscillatory activity plays a role in the association of odors and the preference for them.
\end{abstract}

The olfactory systems in various animals exhibit oscillatory activity, including the terrestrial slug (Gelperin and Tank 1990), locust (Laurent and Naraghi 1994), frog (Ottoson 1959), turtle (Beuerman 1975), hedgehog (Adrian 1942), rabbit (Adrian 1950), and monkey (Hughs and Mazurowski 1962). However, the roles and functions of these oscillatory activities are not well understood. Hypotheses proposed concerning them include the following: (1) They are a consequence of mechanical stimulation of the olfactory receptors (Adrian 1942); (2) they represent "preferred states of activity" for reinforced odors (Freeman and Grajski 1987); (3) odors are encoded by different oscillation modes (Li and Hopfield 1989); (4) they are related to encoding of the intensity of sensory stimuli (Hopfield 1995); (5) they are related to representation of stimuli by the firing sequences of neural assemblies (Wehr and Laurent 1996); and (6) they play roles in the mechanisms of fine odor discrimination (Stopfer et al. 1997; Teyke and Gelperin 1999).

For terrestrial slugs, olfactory information is the most important means of recognizing the external world, and slugs exhibit various types of odor learning (Sahley et al. 1981, 1990; Hopfield and Gelperin 1989; Suzuki et al. 1994). Slugs have two pairs of tentacles that sense odors via olfactory receptors at their tips. Odor information is transferred to the procerebral (PC) lobe, which is the lateral part of the cerebral ganglion and is specialized for olfactory information processing in terrestrial slugs and snails (Ratte and Chase 1997; Chase 2000; Zaitseva 2000). On the surface of the PC lobe, large numbers of cell bodies of interneurons are arranged and elongate their neuropils toward the inside of the PC lobe, where afferent fibers from the tentacles are projected (Kawahara et al. 1997). The PC exhibits oscillation of local field potentials (LFP) at $\sim 0.7 \mathrm{~Hz}$ (Gelperin and Tank 1990). It is thus suitable for study of the contribution of oscillation to information processing in a nervous system that features

${ }^{4}$ Corresponding author.

E-mail sekiguchi@jp.honda-ri.com; fax 81-48-4625221.

Article is online at http://www.learnmem.org/cgi/doi/10.1101//m.1867110. learning, and has been studied extensively (for reviews, see Gelperin 1999, 2006; Watanabe et al. 2008). For example, LFP oscillation is synchronized over the entire PC lobe, with wave propagation from apical to basal parts (Delany et al. 1994; Kawahara et al. 1997; Kimura et al. 1998c). The application of learned odors changes the frequency of the LFP oscillation (Kimura et al. 1998a; Inoue et al. 2006). In addition, when a fluorescent dye, Lucifer Yellow (LY), is injected into the body cavity after odor learning, some of the PC interneurons incorporate the dye (Kimura et al. 1998b; Gelperin 1999; Ermentrout et al. 2001). Since the incorporation of LY depends on conditioning treatment and the duration between conditioning and LY injection (Kimura et al. 1998b), LY-labeled neurons are related to the formation of memory of learned odors. Although several computational models have been proposed to describe the behaviors of PC oscillation including learning-dependent LY labeling (Yamada et al. 1997; Ermentrout et al. 1998, 2001, 2004), no model deals with odor-taste association.

In this study, the relationships between conditioning strategies (appetitive or aversive) and LY labeling were investigated. Then, a simple model based on nonlinear oscillators was constructed to account for the results of the present and previous studies. Based on experimental findings and calculations, the contribution of oscillatory activity to the odor-taste-learning process is then discussed.

\section{Results}

\section{Conditioned behaviors}

Figure 1 shows the combined results of one-trial aversive and appetitive conditioning to carrot and cucumber odors. With aversive conditioning, the proportion of "eat" slugs significantly decreased while that of "avoid" slugs significantly increased (control vs. aversive: $P<0.01, \chi^{2}$ test). In the same way, the proportion of "eat" slugs was significantly higher in the appetitive group than in the control group (control vs. appetitive: $P<0.05, x^{2}$ test). These results show that the conditioning procedure used was effective. 


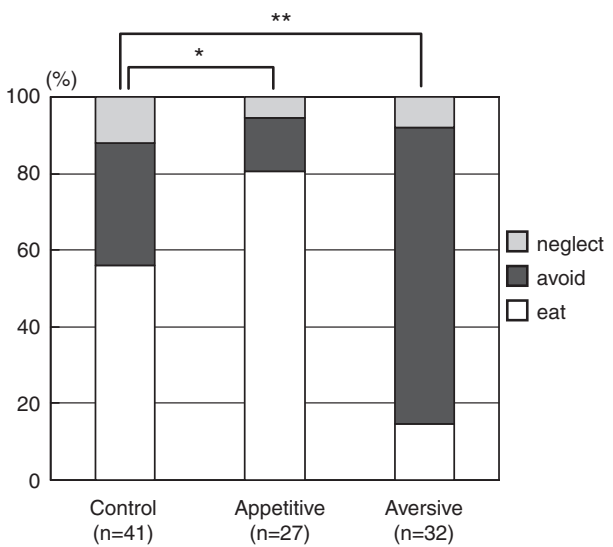

Figure 1. Conditioned behaviors of slugs. Slugs received the conditioning or control treatment only once. The behavior after conditioning was categorized as "eat," "avoid," or "neglect," and the "neglect" slugs were omitted from statistical testing. Results of the experiments using carrot and cucumber odors as CSs were combined. $\left({ }^{*}\right) P<0.05,\left({ }^{* *}\right)$ $P<0.01$ by $\chi^{2}$ test. (n) Number of slugs.

\section{LY uptake}

When LY was injected into the slug body cavity $20 \mathrm{~min}$ after conditioning, a cluster of PC interneurons which incorporated LY (Fig. 2) was observed. Tables 1 and 2 show the details of LY-labeled neurons. As reported by Kimura et al. (1998b) and Ermentrout et al. (2001), a cluster of LY-labeled neurons was observed in one side of the PC lobe. Several LY-labeled neurons were observed in the PC lobe contralateral to the PC lobe classified as "labeled" and in the PC lobes classified as "unlabeled," though in these cases the LY-labeled neurons did not form a cluster (Table 1). As shown in Table 2, the probability of LY labeling was higher in conditioned than in control slugs. In addition, the probability and the average number of LY-labeled neurons did not depend on the CS odor. However, LY labeling was more frequently observed in the aversively trained slugs ( 21 of 38 slugs) than in the appetitively trained slugs (13 of 40 slugs). In addition, clusters of LY-labeled neurons along the dorsal-ventral axis, the "d-v belt" (Fig. 2B), were observed in six PC lobes in both the aversively and appetitively trained slugs. Interestingly, a cluster along the apical-basal axis, the "a-b belt" (Fig. 2C), was observed in one aversively trained slug and three appetitively trained slugs.

The average relative position of LY-labeled neurons in the PC lobe was calculated for each slug (Fig. 3A) and plotted as shown in Figure 3B. The dependence of location of the LY-labeled cluster on CS odor (carrot/cucumber) and conditioning strategy (aversive/appetitive) was examined by two-way ANOVA. A significant difference was observed only in the apical-basal position of LY-labeled clusters between the aversively and appetitively trained slugs (Fig. 3B, upper panel, Ave vs. App: $F=9.23, P<$ 0.01 ; Ca vs. $\mathrm{Cu}: F=0.12, P>0.5$; right panel, Ave vs. App: $F=$ $0.60, P>0.4$; Ca vs. Cu: $F=0.38, P>0.5$ ), showing that the position of LY-labeled clusters was more apical in the appetitively trained slugs than in the aversively trained slugs (see details in the legend to Fig. 3).

Since LY-labeled neurons were rarely observed in the control slugs, it can be expected that LY labeling accompanies memory formation or a process of consolidation. In addition, the present findings suggest that the position of the LY-labeled neurons along the basal-apical axis may indicate an internal representation of the learned odor preference of each slug.

\section{Conceptual model of PC lobe}

A simple computational model was constructed based on results of previous studies of the PC lobe to account for the present experimental findings. As reported by Gelperin and Tank (1990), the PC lobe exhibits oscillatory activity, as also observed in other olfactory systems. The model therefore consisted of a network of nonlinear oscillators. The experimental results taken into consideration in this model were as follows:

(Observation 1) Two oscillatory activities were observed in the PC lobe. One was observed from the cell layer of the PC lobe (Gelperin and Tank 1990) and the other from the neuropil layer (Iwama et al. 1997).

(Observation 2) The propagating wave from the apical to the basal area was observed in the cell body layer (Delany et al. 1994). In addition, oscillatory activity was observed even in an isolated piece of the PC lobe. The frequency of oscillation in the apical pieces was higher than that in the basal pieces (Ermentrout et al. 1998).

(Observation 3) Olfactory information received at the two pairs of tentacles is transferred to the PC lobe, mesocerebrum (MsC), and metacerebrum (MtC) (Kimura et al. 1998b). Taste information received at the lip is transferred to $\mathrm{MsC}$ and $\mathrm{MtC}$ but not directly to the PC lobe (T Kimura, unpubl.).

(Observation 4) A blocker of gap junction, 18- $\alpha$-glycerrhetinic acid, decreased oscillatory local field potentials (Ermentrout et al. 2004).

Based on these observations, we constructed the computational model shown in Figure 4. The model consisted of two layers of nonlinear oscillators, van der Pol (VDP) oscillators, with 20 units in each layer. Layers 1 and 2 correspond to the cell body and neuropil oscillators, respectively (Observation 1 ). Since the PC lobe exhibits oscillatory activity with no phase delay along the dorsalventral axis (Kimura et al. 1998c), each layer was collapsed to a single onedimensional chain of oscillators. There is a gradient in the intrinsic frequency of the oscillators (apical > basal, Observation 2), and each oscillator is connected with neighboring oscillators with interaction mimicking an electrical synapse (Observation 4). Odor input modeled as I is transferred to layer 1 oscillators and taste input is hypothesized to evoke "preference" at MsC or MtC (Observation 3). The taste preference is modeled as changing $\mathrm{K}_{2, \mathrm{j}}$ in the way that aversive and appetitive tastes
Figure 2. Examples of LY-labeled PC neurons (arrows). (A) Posterior surface view of PC lobe after aversive conditioning to cucumber odor. $(B, C)$ Appetitive conditioning to carrot odor. Bars, $100 \mu \mathrm{m}$. 
Table 1. Statistical comparison of numbers of slugs with PC lobes classified as "labeled" or "unlabeled"

\section{Labeled PC ${ }^{\mathrm{a}}$}

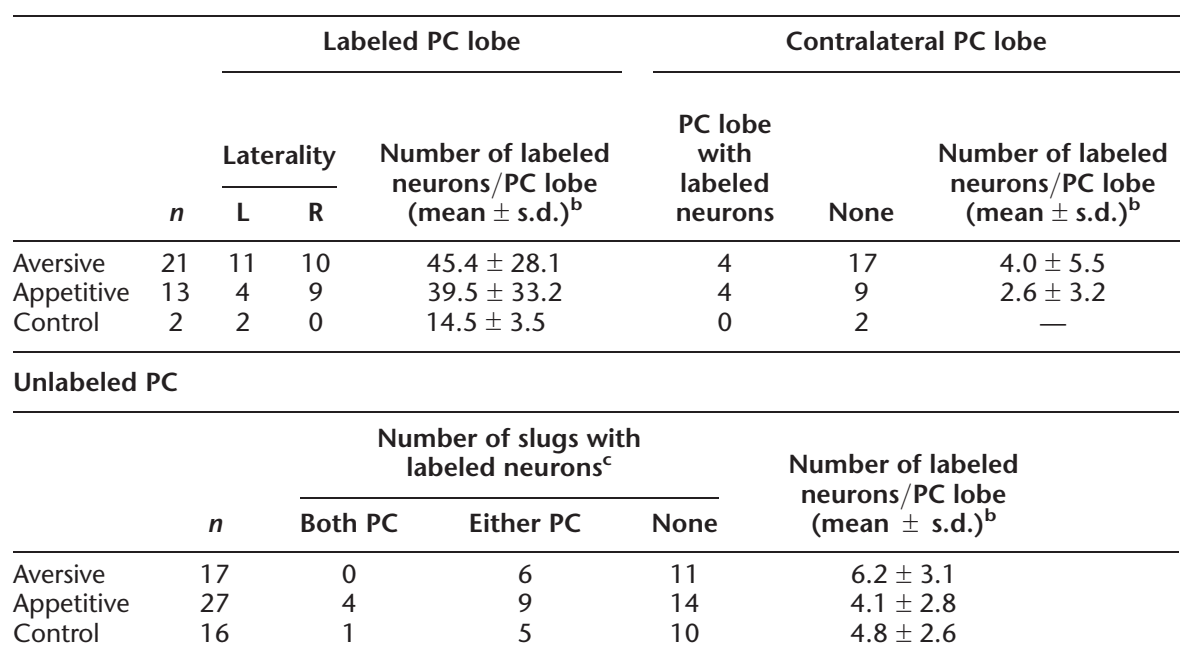

aLaterality indicates the side of the labeled PC lobe; the PC lobe of the other side is considered "contralateral."

${ }^{\text {b}}$ Numbers were obtained by averaging the PC lobes having one or more labeled neurons.

' (Both PC) Slugs with any LY-labeled neurons in both PC lobes; (either PC) slugs with LY-labeled neurons in one PC lobe; (none) slugs with no LY-labeled neurons.

decrease and increase intrinsic frequency of layer 2 oscillators, respectively. Details of the model are provided in the Materials and Methods section.

\section{Basic model behaviors}

The behavior of the model PC lobe without input signal is shown in Figure 5A. The entire network oscillates with a single frequency and the phase in the apical part advances to that in the basal part, which corresponds to the wave propagating from the apical to the basal part of the PC lobe (Gelperin and Tank 1990), as expected from the calculation by Yamada et al. (1997). We checked model behaviors in the following conditions for which behaviors of the biological PC lobe were reported (Ermentrout et al. 1998).

\section{Slice cut across wave axis}

The model was constructed taking this behavior into consideration (Observation 2). Actually, when both $\mathrm{e}_{1}$ and $\mathrm{e}_{2}$ between units 10 and 11 are zero, the apical half oscillates with higher frequency than the basal half (Fig. 5B).

\section{Cutting cell layer}

In the real PC lobe, cutting a part of the cell layer results in increase in apical-basal phase delay. In the model, this treatment corresponds to the condition in which $\mathrm{e}_{1}$ between units 10 and 11 is zero. Coupling of the entire PC lobe remains and the frequency stays the same. However, there is a gap in the propagating wave between units 10 and 11, and the apical-basal phase delay increases slightly (Fig. 5C).

\section{Cutting neuropil layer}

In the real PC lobe, the apical-basal coupling remains after cutting of the neuropil layer. When $e_{2}$ between units 10 and 11 is zero, results similar to those in the case of cutting of the cell layer are obtained. However, there is no gap in wave propagation (Fig. 5D).

\section{Isolated cell body layer}

The actual isolated cell layer still exhibits wave propagation. The model also exhibits wave propagation when $\mathrm{e}=0$. In addition, the frequency is decreased and the apicalbasal phase delay is increased (Fig. 5E).

\section{Odor stimulation}

In the real PC lobe, odor input decreases the apical-basal phase delay. When a stimulus was applied to layer 1 of the model $(I=0.002)$, both the frequency and the phase delay transiently increased and then decreased (Fig. 5F). Recovery from these changes occurred after stimulation. Larger input resulted in less apical-basal phase delay (data not shown).

These findings indicate that the behaviors of the present model mimic those of the biological PC lobe well.

\section{Model behaviors during conditioning}

When preference input (evoked by taste) is applied, which corresponds to change in the intrinsic frequencies of layer 2 oscillators, the entire network still exhibits coupling; however, the frequency changes (Fig. 6). Appetitive (smaller $\mathrm{K}_{2, \mathrm{j}}$ ) and aversive (larger $\mathrm{K}_{2, \mathrm{j}}$ ) input increase and decrease the frequency, respectively. This mimics the behavior of the real PC lobe well, in which conditioned odor input can alter the frequency (Kimura et al. 1998a). The phase differences between the corresponding units of layers 1 and 2 during this process are shown in Figure 7A. As the intrinsic frequencies of layer 2 units decrease $\left(K_{2, j}\right.$, are increased), the position-phase relation curves shift in the basal direction. This indicates that the position of a certain interlayer phase difference (for example, the position with no phase difference) depends on preference input. Figure $7 \mathrm{~B}$ shows the position-phase relation

Table 2. Statistics of LY labeling

\begin{tabular}{lccc}
\hline & \multicolumn{2}{c}{ Number of slugs } & \\
\cline { 2 - 3 } & $\begin{array}{c}\text { Labeled/ } \\
\text { Tried }\end{array}$ & $\begin{array}{c}\text { Patch/d-v } \\
\text { belt } / \mathbf{a}-\mathbf{b} \text { belt }\end{array}$ & $\begin{array}{c}\text { Number of labeled } \\
\text { neurons (mean } \pm \text { s.d.) }\end{array}$ \\
\hline Carrot & $10 / 20$ & $6 / 4 / 1$ & $42.7 \pm 30.7$ \\
$\quad \begin{array}{c}\text { Aversive } \\
\text { Appetitive }\end{array}$ & $9 / 21$ & $2 / 5 / 2$ & $49.0 \pm 36.1$ \\
$\begin{array}{c}\text { Control } \\
\text { Cucumber }\end{array}$ & $1 / 9$ & $1 / 0 / 0$ & 12 \\
$\quad$ Aversive & $11 / 18$ & $9 / 2 / 0$ & $43.5 \pm 26.6$ \\
Appetitive & $4 / 19$ & $2 / 1 / 1$ & $18.0 \pm 6.7$ \\
Control & $1 / 9$ & $1 / 0 / 0$ & 17 \\
Total & & $15 / 6 / 1$ & $45.4 \pm 28.1$ \\
Aversive & $21 / 38$ & $15 / 3$ & $39.5 \pm 33.2$ \\
Appetitive & $13 / 40$ & $4 / 6 / 3$ & $14.5 \pm 3.5$ \\
Control & $2 / 18$ & $2 / 0 / 0$ & \\
\hline
\end{tabular}

Seventy-eight slugs were divided into four groups according to the conditioning strategy, aversive or appetitive, and the conditioned odor stimulus, carrot or cucumber. Clusters of LY-labeled neurons were categorized as "patches," "d-v belts," or "a-b belts" based on criteria described in the Materials and Methods. Numbers of LY-labeled neurons were obtained from slugs that exhibited labeling of more than 10 neurons. 
A

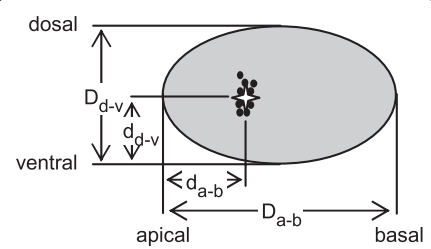

B

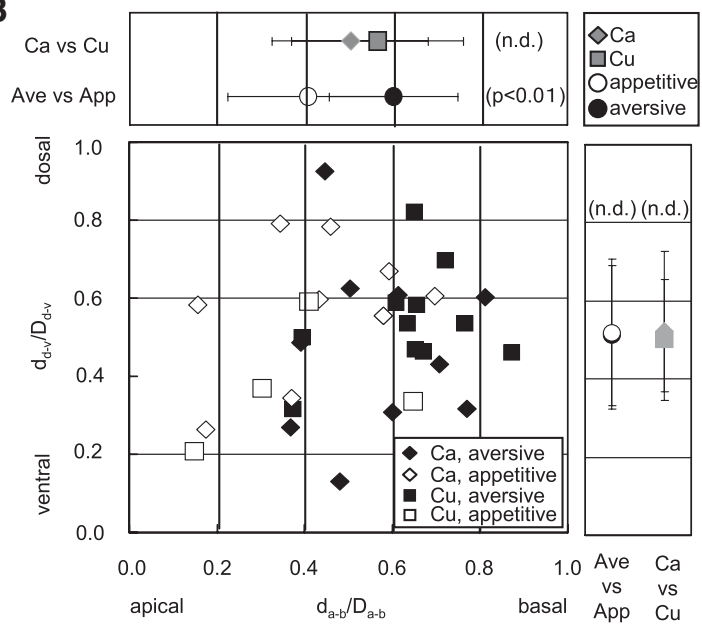

Figure 3. Distribution of LY-labeled neurons. $(A)$ Method used to determine the relative positions of LY-labeled neurons. Each PC lobe was approximated by an ellipse with the major axis corresponding to the apical-basal axis, and the relative position of each LY-labeled neuron was calculated. The averaged position (cross) was used as the representative position of each slug. (B) Averaged relative positions of LY-labeled neurons in each slug. Horizontal and vertical axes are the relative positions in the apicalbasal and ventral-dorsal axes in the PC lobe, respectively. (Diamonds and squares) Position of LY-labeled neurons in the PC from slugs that received conditioning with carrot and cucumber odors as CSs, respectively. (Open and closed marks) Conditioning strategies: (open) appetitive, (closed) aversive. (Upper and right small graphs) Grand averages and standard deviations of the relative positions compared among CSs and conditioning strategies, respectively. Only the apical-basal position significantly differed between appetitively and aversively trained slugs.

the $\mathrm{K}_{2, \mathrm{j}}-\mathrm{P0}$ relationship is maintained. Thus, PO depends on strength of odor input (I), change in frequency due to odor input $\left(\mathrm{K}_{1, \mathrm{j}}\right)$, and preference input $\left(\mathrm{K}_{2, j}\right)$. More specifically, both decrease in $K_{1, j}$ and increase in $K_{2, j}$ shift P0 more basally and the increase in I first shifts PO to the basal region and then to the apical region. To account for the contribution of these parameters to P0, we hypothesized that stronger odor input is accompanied by larger increase in the frequency of the network, and represented the relation of these parameters as $I=0.003 \times\left(2-K_{1,1}\right)$. The results of calculation are shown in Figure 8C. As $\mathrm{K}_{1,1}$ decreases, accompanied by increase in frequency and strength, P0 moves to the basal region but then moves to the apical region. Finally, P0 will reach units 11-13 irrespective of the value of $K_{2, j}$. This results from the combination of contradictory effects of input strength, which shifts P0 position to the apical region, and $K_{1, j}$ which shifts P0 toward the basal region. As a result, $\mathrm{PO}$ is distributed in the basal region when $\mathrm{K}_{2, \mathrm{j}}$ is larger and in the apical region when $\mathrm{K}_{2, \mathrm{j}}$ is smaller. when there is odor input $(\mathrm{I}=0.003)$. In this case, as expected from Figure $5 \mathrm{~F}$, the wave form is distorted and the slope of the curve decreases as $\mathrm{K}_{2, \mathrm{j}}$ is decreased, although it is still the case that the position of a certain phase difference shifts toward the basal region as $\mathrm{K}_{2, \mathrm{j}}$ is increased. In Figure $8 \mathrm{~A}$, the position at which the phase difference is nearest to 0 (P0) is plotted against the strength of odor input as it increases with changing preference input. As the external odor input increases, P0 also moves to the basal region but then moves to the apical region for larger input. However, the $\mathrm{K}_{2, \mathrm{j}}-\mathrm{P0}$ relationship is maintained irrespective of the strength of odor input. As a result, $\mathrm{P0}$ is restricted to the apical region with smaller $\mathrm{K}_{2, \mathrm{j}}$ (appetitive) and is distributed in the basal region with larger $\mathrm{K}_{2, \mathrm{j}}$ (aversive).

Inoue et al. (2006) reported that odor stimulation increased the frequency of LFP oscillation, suggesting that frequency change in layer 1 oscillators will also contribute to behaviors of the model. However, as shown in Figure 8A (right column), our model, which is based on VDP oscillators, does not exhibit an increase in frequency with odor stimulus, I. In order to investigate effects of frequency change in layer 1 oscillators on the behaviors of the model, the parameters affecting the frequency of layer 1 oscillators, $K_{1, j} \mathrm{~s}$, were altered such that $\mathrm{K}_{1,1}$ changed from 0.4 to 2.4 while maintaining the gradient $\mathrm{K}_{1, \mathrm{j}}=\mathrm{K}_{1,1}+0.1(\mathrm{j}-1)$. Figure $8, \mathrm{~A}$ and $B$, shows $K_{1, j}$ vs. PO and $K_{1, j}$ vs. frequency relationship with change in $K_{2, j}$, respectively. As $K_{1, j}$ decreases, the frequency of the entire network increases (Fig. 8B, right column) and $\mathrm{PO}$ moves to the basal region (Fig. 8B, left column). In addition, See details in text.

\section{Discussion}

Learning-dependent LY incorporation in the olfactory system of Limax was first reported by Kimura et al. (1998b) in the case of aversive conditioning, followed by Gelperin (1999) and Ermentrout et al. (2001). Kimura et al. (1998b) showed that a cluster of LY-labeled neurons was observed in the PC lobe of aversively conditioned slugs but was rarely observed in that of control slugs. They also reported that the probability of labeling decreased when the duration from the conditioning to LY injection become longer, and that aversive conditioning to two odors sometimes resulted in two clusters. These findings suggest that it is likely that the LY-labeled neurons contribute to odor-taste associative learning or encode some of the effects of such learning.

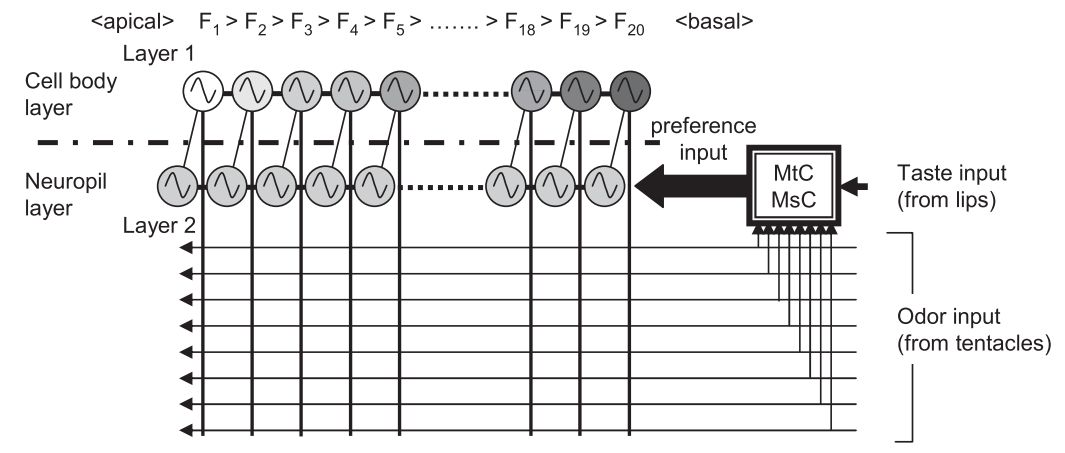

Figure 4. Outline of the model. The model consists of two layers of 20 oscillators each with electrical synaptic interaction within each layer and between layers. There is a gradient in the intrinsic frequency of layer 1 oscillators, higher in the apical and lower in the basal oscillators. The intrinsic frequencies of layer 2 oscillators are the same. Odor signals are inputted to layer 1 oscillators and MtC/MsC. Taste signals are inputted to $\mathrm{MtC} / \mathrm{MsC}$, which evokes a "preference" which is sent to all layer 2 oscillators. 

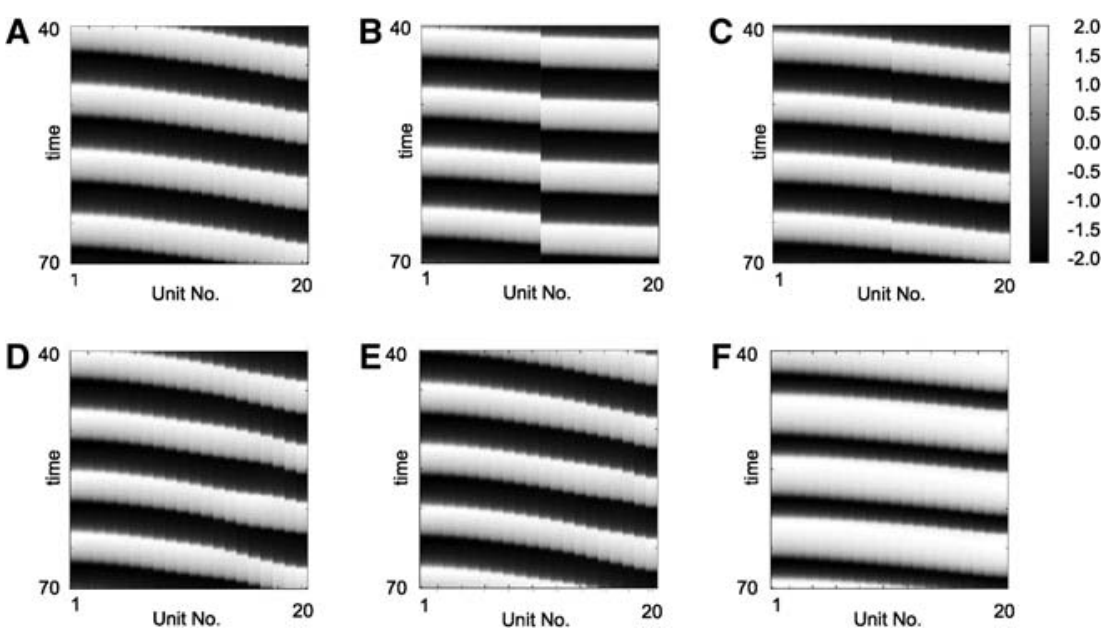

Figure 5. Basic behaviors of the model. Behaviors of layer 1 are shown. Time scales are in arbitrary units, since only relative times are important. Because the van der Pol oscillator oscillates between -2 to 2 , the amplitude is also arbitrary. $(A)$ Wave propagation from the apical to basal region. Parameters are $\mathrm{K}_{1, \mathrm{j}}=2.0+0.1(\mathrm{j}-1), \mathrm{K}_{2, \mathrm{j}}=1.8, \mathrm{e}_{1}=\mathrm{e}_{2}=0.005, \mathrm{e}=0.005$. These parameters are the same in $B-F$ unless otherwise mentioned. $(B)$ Effect of transverse section of PC. This is mimicked by setting of $e_{1}$ and $e_{2}$ between units 10 and 11 to zero. The basal half oscillates with lower frequency. (C) Effect of cutting through cell body layer. In this case, only $\mathrm{e}_{1}$ between units 10 and 11 is zero. $(D)$ Effect of cutting neuropil layer. $e_{2}$ between unit 10 and 11 is zero. (E) Behavior of the isolated cell body layer. In this case $\mathrm{e}=0 .(F)$ Behavior of PC lobe during odor stimulation. I $=0.002$.

According to Kimura et al. (1998b), 65\% of aversively conditioned slugs exhibited LY-labeled PC neurons, forming "patch" or "belt" clusters, and 50\% of slugs exhibited a single "belt" cluster parallel to the dorsal-ventral axis on one side of the PC lobe. Ermentrout et al. (2001) observed LY labeling in $62 \%$ of aversively conditioned slugs and in $27 \%$ of "belt" clusters. In this study, the proportion of aversively trained slugs exhibiting a cluster of LY-labeled neurons was similar, at 55\% (21 of 38 slugs), and a "belt" was observed in $16 \%$ of slugs (six of 38 slugs). The proportion of "belt" clusters was slightly lower than that in previous studies, but still showed that LY labeling occurred in a similar fashion in the present study. In particular, in the case of appetitive conditioning, the probability of observing LY-labeled PC neurons was lower (33\%, 13 out of 40 slugs) than that in aversive slugs. This low probability may have been partly due to the low rate of success of the conditioning, since it can be calculated from Figure 1 that $21.5 \%$ of the slugs changed preference to CS odors in appetitive conditioning, which is much lower than $48.1 \%$ in aversive conditioning. The fact that carrot and cucumber odors are natively appetitive stimuli may contribute to the low success rate of

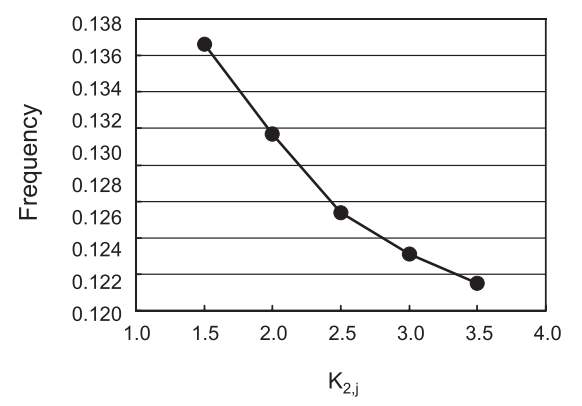

Figure 6. Preference input changes the frequency of $P C$. As $K_{2, j}$ is increased, which corresponds to decrease in the intrinsic frequency of layer 2 oscillators, the frequency of PC decreases. Parameters other than $\mathrm{K}_{2, \mathrm{j}}$ are the same as in Figure 5A.
The most important feature of LY labeling is that the LY-labeled neurons formed a cluster irrespective of its shape, "patch" or "belt." In the PC lobe, afferent fibers from the tentacles enter the PC lobe from the basal part to the apical part. This layer includes the neurites of nonbursting (NB) neurons, which form a dense interacting matrix (Kawahara et al. 1997). The afferent fibers carry chemical features of an odor. Thus, the PC neurons distributed along the apical-basal axis have equal capacity to code information of an odor. Ermentrout et al. (2001) was able to explain single "stripe" formation by a winner-takes-all mechanism of lateral inhibition among NB neurons. However, this mechanism cannot explain the finding of the present study that the position of the LY-labeled cluster in the apical-basal axis depended on the learned preference to CS odors. Thus, an additional mechanism is needed to account for how the position of LY-labeled neurons is determined in the uniform matrix of the afferent fibers and NB neurons.

The model proposed in this study (Fig. 4) provides one possible answer. The structure of the model is similar to that of the model proposed by Ermentrout et al. (1998), in which the oscillators are assigned to bursting (B) and NB neurons and are expressed by phase equations. In addition, they assumed that the activity of NB neurons is tightly locked to and oscillates in the same phase as that of the B neurons at the same spatial point. On the other hand, the model in this study focuses on interaction between two oscillatory activities in the PC lobe, one in the cell body layer (Gelperin and Tank 1990) and the other in the neuropil layer (Iwama et al. 1997). Thus, the oscillators are described independently and are connected by electrical synapse-like interaction. This allows us to study the phase relation among oscillators, and the present model mimics the behaviors of the biological PC lobe as well as that of Ermentrout et al. (1998).

Aversive and appetitive conditioning involve association of CSs with preferences (values). This means that information about CSs and preference are independently supplied. In the slug, odor inputs (CSs) are transferred to the $\mathrm{PC}$ lobe and $\mathrm{MsC} / \mathrm{MtC}$, and taste inputs to $\mathrm{MsC} / \mathrm{MtC}$ but not the PC lobe. In addition, PCablated slugs fail to avoid aversively conditioned odors but not 
A

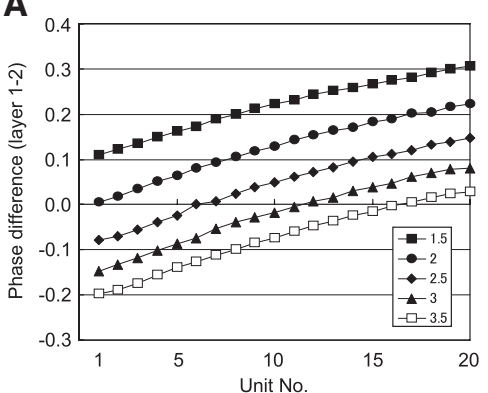

B

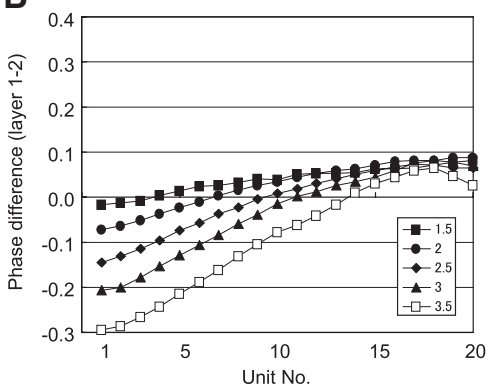

Figure 7. Effect of preference input on the phase difference between oscillators of the same position. $(A)$ Phase difference when only the preference input exists. $\mathrm{K}_{2, \mathrm{j}}$ is altered from 1.5 to 3.5. (B) Phase difference when both the preference input and the odor input exist $(I=0.003)$. Other parameters are the same as in Figure 5A.

innately aversive odors (onion or garlic) (Kasai et al. 2006). Also, innately aversive taste (quinidine) evokes avoidance behaviors. Based on these findings, we hypothesized in the present model that the PC lobe receives odor information directly from the tentacles and preference information from $\mathrm{MsC} / \mathrm{MtC}$, where preference information is evoked from odor or taste information. This hypothesis is supported by the experimental findings that some neurons in MtC contain serotonin (Shirahata et al. 2004) and that serotonin alters the frequency (Gelperin et al. 1993) and wave propagation (Iwama et al. 1999) of PC oscillation. According to an optical recording study by Makinae et al. (2008), stimulation of tentacle nerves first evokes activity in $\mathrm{MtC}$ followed by that in PC, a finding which also supports our hypothesis.

Our model suggests that memory of odor-taste conditioning is formed in the following way. Odor input is transferred to the PC and $\mathrm{MsC} / \mathrm{MtC}$. Taste input is transferred to $\mathrm{MsC} / \mathrm{MtC}$ and evokes preference information, which is then sent to the PC. The preference input changes the frequency of oscillators in the neuropil layer, which alters the phase difference between oscillators of the same spatial position in the cell layer and neuropil layer. A certain phase difference functions like a "gate" and allows odor information to be stored with its preference at the position of phase difference. For example, as oscillatory units in both cell layer and neurophil layer oscillate in a same phase at the P0, neurons in the cell layer (PC interneurons) at the position would be more activated than those at the other positions. This enables synaptic strength between the PC interneurons and neurites projected from the tentacles to increase. A winner-takes-all mechanism such as lateral inhibition among PC interneurons, which is suggested by Ermentrout et al. (2001), will help to enhance the contrast of the activities. This will result in memory formation in restricted area. Once the synaptic change occurred, a conditioned odor can activate these neurons, which was observed in an optical recording study by Kimura et al. (1998c). To explain these findings based on the results of calculation (Fig. 8C), suppose that $K_{1,1}=2.0$ and $K_{2, j}=1.8$ is at resting state (frequency $=$ 0.132 ), and the "gate" will be open at the position of no phase difference (P0). In this condition, no P0 is found at the resting state. Odor input (for example, $\mathrm{K}_{1,1}=1.4$ ) shifts P0 to unit 16 . However, when aversive input exists (increase $K_{2, j}$ to 2.2), P0 is unit 19 and frequency $=0.133$. Similarly, with appetitive input (decrease $K_{2, j}$ to 1.4), P0 becomes unit 7 and frequency $=0.139$. Thus, under conditions in which the frequency is increased as described by Inoue et al. (2006), it is possible to realize the representation of odor preference along the apical-basal axis of the PC lobe.

In this model, odors are represented as patterns of activation of neurons projected from tentacles to the PC lobe, and only the
$\mathrm{K}_{1, j} \mathrm{~s}$ change in similar fashions to both carrot and cucumber odors and that P0 shifts mainly as a function of preference input, $\mathrm{K}_{2, \mathrm{j}}$. As a result, $\mathrm{PO}$ shifts to the apical or basal region for appetitive (smaller $\mathrm{K}_{2, \mathrm{j}}$ ) or aversive (larger $\mathrm{K}_{2, \mathrm{j}}$ ) preference input, respectively. The model can also explain the "a-b belt" cluster observed mainly in appetitively trained slugs (Fig. 2C). As shown in Figure 7B, with odor input, the slope of the position-phase difference curve becomes very small when $\mathrm{K}_{2, \mathrm{j}}$ is small (appetitive conditioning). This may enable the "gate" to open along a longer apical-basal distance, resulting in an "a-b belt" cluster.

This model should be checked by electrophysiological examination of the phase relationship between oscillations recorded from the PC surface and from the neuropil layer. Specifically, a phase difference between surface and neuropil oscillations should be detected, and the position with the phase difference should shift to the apical or basal region when appetitive or aversive odor/taste stimuli are applied, respectively. In addition, the shift will differ between an isolated PC lobe and a whole-brain preparation, since the former lacks preference input from MtC/ MsC while the latter possesses it.

Oscillatory activity is a common feature of olfactory systems and has various potentials for the representation or processing of information. For example, Freeman and Grajski (1987) proposed that odor information is represented as the spatial pattern of oscillation "amplitude" in the rabbit olfactory bulb. Kimura et al. (1998a) showed that the change in "frequency" of oscillation represents odor preference. Li and Hopfield (1989) hypothesized encoding and distinguishing of odors by different "oscillation modes" based on their computational model. Finally, Laurent and Naraghi (1994) proposed that "temporal evolution" of oscillation encodes odor information in the locust olfactory system. In other brains, such as that of the cat, the formation of "coherent states" resulting from entrainment among neurons is thought to play roles in visual information processing (Gray et al. 1989). In addition, the time of firing of hippocampal place cells in relation to theta band oscillation has been well studied, and firing "phase" could be a powerful means of representing absolute position (Jensen and Lisman 2000), relative position in a place field (O'Keefe and Recce 1993; Huxter et al. 2003), sequences (Skaggs et al. 1996; Foster and Wilson 2007), and movement trajectories (Dragoi and Buzsaki 2006). Thus, theta band oscillation in the hippocampus is also of interest in the study of the roles of oscillatory activity in the brain. It was recently reported that the oscillations in area CA1 are traveling waves along the septo-temporal axis of the hippocampus, and that spiking of CA1 pyramidal cells is modulated by such waves (Lubenov and Siapas 2009). The findings of the present study suggest another possible contribution of "phase" to information processing in the brain 
A
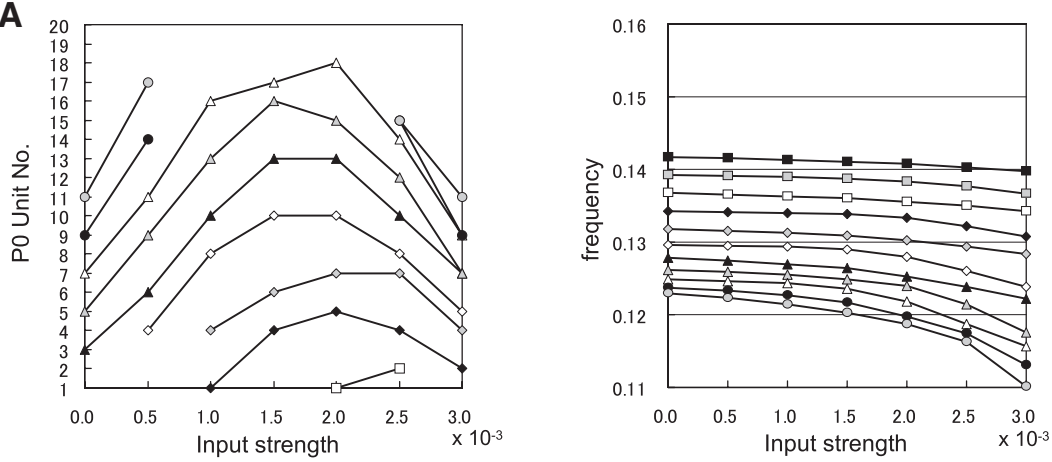

B
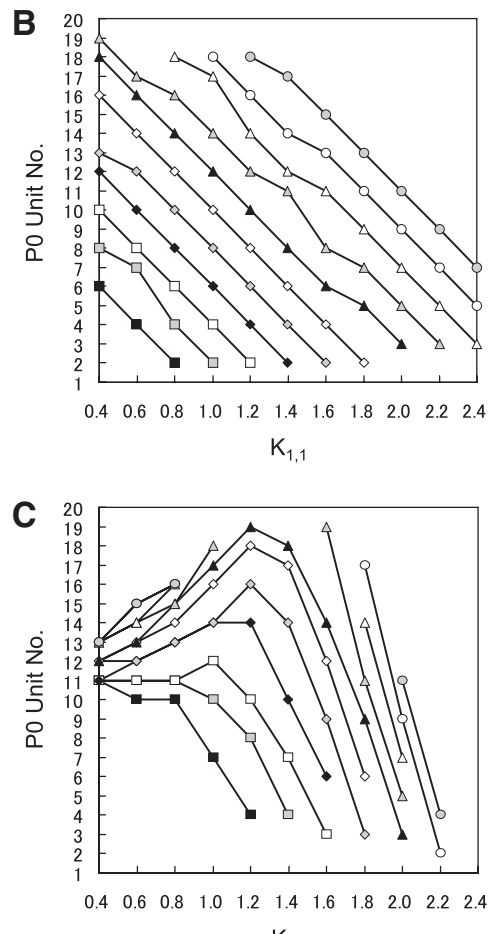

$\mathrm{K}_{1,1}$
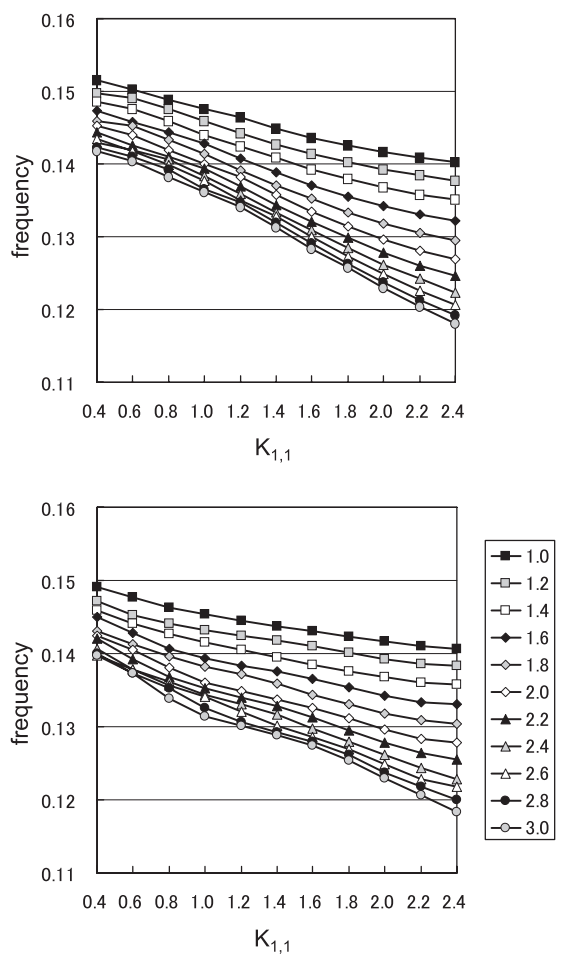

Figure 8. $P 0$ shifts and frequency of $P C$ lobe oscillation in relation to $K_{2, j}, I$ and $K_{1, j}$. $(A)$ Effect of input strength (I). (B) Effect of intrinsic frequency $\left(\mathrm{K}_{1, j}\right)$. (C) Combined effects of $\mathrm{I}$ and $\mathrm{K}_{1, \mathrm{j}}$ with relationship $\mathrm{I}=$ $0.003\left(2-\mathrm{K}_{1,1}\right)$. (Left) Effects of changes in parameters on P0. (Right) Effects of changes in parameters on the frequency. Different marks correspond to differences in preference input $\left(K_{2, j}\right)$ from 1.0 to 3.0. Other parameters such as connections among units are the same as Figure 5.

involving memory formation. This is not surprising, since spike timing-dependent plasticity (Markram et al. 1997) can be interpreted as a type of phase-dependent memory formation under theta oscillation. Further physiological studies are needed to elucidate the origin of neuropil oscillators and their contributions to odor information processing.

\section{Materials and Methods}

\section{Animals}

Terrestrial slugs (Limax marginatus) were raised in the laboratory on rat chow (CRF-1, Charles River Laboratories International, Inc., Japan) at $20^{\circ} \mathrm{C}$. Two or $3 \mathrm{~d}$ before the start of conditioning, 2 - to 4-mo-old animals (1.0-1.5 g) were individually placed in plastic containers $(113 \times 105 \times 28 \mathrm{~mm})$ lined with moistened filter paper and starved until the start of experiments.
Stimuli and solutions

Carrot juice and cucumber juice were used as conditioned stimuli (CSs). Carrots were homogenized using a blender without dilution and filtered through filter paper. The obtained carrot juice was divided into 5-mL aliquots in disposable tubes and stored in a freezer at $-20^{\circ} \mathrm{C}$. The cucumber juice was obtained in the same fashion. A saturated solution of quinidine sulfate in slug saline $(70 \mathrm{mM}$ $\mathrm{NaCl}, 2 \mathrm{mM} \mathrm{KCl}, 4.9 \mathrm{mM} \mathrm{CaCl}_{2}, 4.6 \mathrm{mM}$ $\mathrm{MgCl}_{2}, 5.0 \mathrm{mM}$ glucose, $4.3 \mathrm{mM}$ HEPES, and $5.7 \mathrm{mM}$ HEPES-Na, pH 7.4) was used as an unconditioned stimulus (US).

An activity marker, $25 \mathrm{mg}$ of Lucifer Yellow (LY) dilithium salt (MP Biomedicals, LLC), was diluted to $2 \mathrm{~mL}$ with slug saline. The LY solution was divided into $100-\mu \mathrm{L}$ aliquots and stored in a freezer at $-20^{\circ} \mathrm{C}$. An anesthetizing saline solution $(55 \mathrm{mM} \mathrm{MgCl} 2,4.3 \mathrm{mM}$ HEPES, and $5.7 \mathrm{mM}$ HEPES-Na, $\mathrm{pH} 7.4$ ) was used to immobilize slugs when isolating the central nervous system.

\section{Conditioning and testing procedures} Slugs in the aversive group (aversively trained slugs) were transferred with forceps from individual containers onto the center of a glass plate $(200 \times$ $200 \mathrm{~mm}$ ) and left for several minutes. After the slug had begun to crawl straight in any direction, a small strip of filter paper $(15 \times 3 \mathrm{~mm})$ immersed with the required CS (carrot or cucumber) was placed in its crawling direction $2-3 \mathrm{~cm}$ from its head. Most of the slugs stopped in front of the filter paper, extended their tentacles, and started to eat the CS (naïve slug's response). Immediately after the slug began to eat, a few drops of US solution were placed on its head. The slugs then secreted mucus to remove the US from the body and crawled in another direction. After slugs had removed the mucus, they were returned to individual containers. The slugs in the appetitive group (appetitively trained slugs) and the control group (control slugs) were treated in the same fashion as the aversively trained slugs until they approached the filter paper. The appetitively trained slugs were allowed to eat the CS. They were returned to individual containers $>1$ min after they had stopped eating and begun to crawl again. The control slugs were returned to individual containers immediately after they started to eat the CS, left for $1 \mathrm{~h}$, placed on a glass plate again, and treated with the US. In all experiments, including LY experiments, each slug received the conditioning or control treatment only once.

The slugs were tested for odor preference $2-3 \mathrm{~h}$ after conditioning. Slugs were placed on a glass plate and an odor source (CS) was presented as in the above conditioning procedures. The slug's behavior in response to CS odors was categorized as follows: (1) Slugs that ate the CS were assigned to the "eat" group; (2) slugs that turned their head and crawled in another direction were assigned to the "avoid" group; and (3) slugs that crawled over the CS were assigned to the "neglect" group. Since it was possible that the slugs assigned to the "neglect" group did not recognize odor in normal fashion, these slugs were omitted from statistical analysis. Slugs assigned to the "neglect" group accounted for 
$9.0 \%$ of all slugs, and there was no statistical difference in the proportion of "neglect" slugs among the groups.

One experiment was carried out with 16-20 slugs with two groups of different combinations among the appetitively trained, aversively trained, and control groups using the same CS, and the results were combined.

\section{LY experiments}

In the LY experiment, slugs were conditioned but not tested for odor preference before or after LY treatment, since the testing procedure itself can contribute to memory formation (Sekiguchi et al. 1997). Seventy-eight slugs were used in this experiment (see Table 1 for details on the groups).

Twenty minutes after the aversive or appetitive conditioning, $100 \mu \mathrm{L}$ of LY solution was injected into the slug's body cavity using a disposable syringe. The slug was kept in the dark for $1 \mathrm{~h}$ and was then anesthetized by injection of the anesthetizing solution. The cerebral and subesophageal ganglia were dissected from the body, washed with the anesthetizing solution several times, and fixed in $4 \%$ paraformaldehyde solution for $>4 \mathrm{~h}$. Each preparation was stored individually in a small glass chamber. After dehydration with a series of ethyl alcohols (60\%-80\%, 95\%-100\%, 5 min each) and clearing with methylbenzoate in the individual chamber, each preparation was placed so that, as far as possible, its posterior surface faced the lens of the microscope. The image was observed and photographed under confocal microscopy (LSM410, Carl Zeiss) by an investigator unfamiliar with the history of the preparation. The number and relative positions of LY-labeled neurons in the PC lobe were determined by the investigator.

To obtain relative positions, the PC lobe was first approximated by an ellipse, with the major axis corresponding to the apical-basal axis. Then the distances from the apical edge $\left(\mathrm{d}_{\mathrm{a}-\mathrm{b}}\right)$ and ventral edge $\left(\mathrm{d}_{\mathrm{d}-\mathrm{v}}\right)$ were measured and divided by the apicalbasal length $\left(D_{a-b}\right)$ and the dorsal-ventral length $\left(D_{d-v}\right)$, respectively (see Fig. 3A). The relative positions of the LY-labeled neurons obtained were averaged and used as the representative position of LY-labeled neurons for each slug. PC lobes with fewer than 10 LY-labeled neurons were categorized as "unlabeled" and omitted from analysis.

The clusters of LY-labeled neurons were categorized as "patches," "d-v belts," or "a-b belts." When the dorsal-ventral to apical-basal length ratio of the cluster was $>2$ or $<0.5$, the cluster was categorized as a "d-v belt" or "a-b belt," respectively. All other clusters were categorized as "patches."

\section{Model}

The model consisted of two layers of nonlinear oscillators with 20 units in each layer. In both layers the unit oscillator is described by a "van der Pol oscillator," as follows:

$$
\begin{aligned}
& \mathrm{dx}_{\mathrm{i}, \mathrm{j}} / \mathrm{dt}=\mathrm{y}_{\mathrm{i}, \mathrm{j}}+\mathrm{G}_{\mathrm{i}}+\mathrm{H}(+\mathrm{I}) \\
& \mathrm{dy}_{\mathrm{i}, \mathrm{j}} / \mathrm{dt}=\mathrm{K}_{\mathrm{i}, \mathrm{j}}\left(1-\mathrm{x}_{\mathrm{i}, \mathrm{j}}^{2}\right) \mathrm{y}_{\mathrm{i}, \mathrm{j}}-\mathrm{x}_{\mathrm{i}, \mathrm{j}}
\end{aligned}
$$

where

i: $\quad$ layer (cell body layer: 1 , neuropil layer: 2 )

$\mathrm{j}: \quad$ unit $(\mathrm{j}=1,2, \ldots, 20)$

$\mathrm{G}_{\mathrm{i}}$ : intralayer interaction

$\mathrm{H}$ : interlayer interaction

I: odor input to layer 1 oscillators

$\mathrm{K}_{\mathrm{i}, \mathrm{j}}$ is a constant, with larger $\mathrm{K}_{\mathrm{i}, \mathrm{j}}(>0)$ values resulting in lower intrinsic frequency in each oscillator. For layer 1 , the $K_{1, j}$ values of the more apical oscillators are smaller than those of the more basal oscillators:

$$
\mathrm{K}_{1, \mathrm{j}}=2.0+0.1(\mathrm{j}-1)
$$

That is, $K_{1,1}=2.0$ and $K_{1,20}=3.9$. For layer $2, K_{2, j}$ was chosen to be the same $\left(K_{2, j}=2.5\right)$ when there was no taste (preference) input. Aversive and appetitive taste input are represented as increase and decrease in $\mathrm{K} 2$, j, respectively. These changes result in decrease and increase in the intrinsic frequencies of layer 2 oscillators. $G_{i}$ was chosen from Observation 4 in the Results section as

$$
G_{i}=e_{i}\left(x_{i, j-1}-x_{i, j}\right)+e_{i}\left(x_{i, j+1}-x_{i, j}\right) .
$$

This mimics an electrical synapse, and $e_{i}$, the strength of the interaction, is the same within each layer. The oscillators at both ends interact only with each neighboring oscillator. $\mathrm{H}$ is also chosen to be an electrical synapse as follows:

$$
\begin{array}{ll}
H=e\left(x_{2, j}-x_{1, j}\right) & \text { for layer } 2 \text { to } 1 \\
H=e\left(x_{1, j}-x_{2, j}\right) & \text { for layer } 1 \text { to } 2
\end{array}
$$

where e is the strength of the interaction and unit $\mathrm{j}$ in layer 1 interacts only with unit $\mathrm{j}$ in layer 2 . Stimulus input I is applied only to layer 1 . This is because afferent fibers from the nose interact with the neuropils of nonbursting (NB) neurons that belong to the cell body layer (Kawahara et al. 1997).

The behavior of the model was calculated using the fourthorder Runge-Kutta-Gill method with a time step of 0.01 . Calculations were performed using MATLAB software.

\section{References}

Adrian ED. 1942. Olfactory reactions in the brain of the hedgehog. J Physiol (Lond) 100: 459-473.

Adrian ED. 1950. The electrical activity of the mammalian olfactory bulb. Electroencephalogr Clin Neurophysiol 2: 377-388.

Beuerman RW. 1975. Slow potentials of the turtle olfactory bulb in response to odor stimulation of the nose. Brain Res 97: 61-78.

Chase R. 2000. Structure and function in the cerebral ganglion. Microsc Res Tech 49: 511-520.

Delany KR, Gelperin A, Fee MS, Flores JA, Gervais R, Tank DW, Kleinfeld D. 1994. Waves and stimulus-modulated dynamics in an oscillating olfactory network. Proc Natl Acad Sci 91: 669-673.

Dragoi G, Buzsaki G. 2006. Temporal encoding of place sequences by hippocampal cell assemblies. Neuron 50: 145-157.

Ermentrout B, Flores J, Gelperin A. 1998. Minimal model of oscillations and waves in the Limax olfactory lobe with tests on the model's predictive power. J Neurophysiol 79: 2677-2689.

Ermentrout B, Wang JW, Flores J, Gelperin A. 2001. Model for olfactory discrimination and learning in Limax procerebrum incorporating oscillatory dynamics and wave propagation. J Neurophysiol 85: $1444-1452$.

Ermentrout B, Wang JW, Flores J, Gelperin A. 2004. Model for transition from waves to synchrony in the olfactory lobe of Limax. JComp Neurosci 17: $365-383$.

Foster DJ, Wilson MA. 2007. Hippocampal theta sequences. Hippocampus 17: 1093-1099.

Freeman WJ, Grajski KA. 1987. Relation of olfactory EEG to behavior: Factor analysis. Behav Neurosci 101: 766-777.

Gelperin A. 1999. Oscillatory dynamics and information processing in olfactory systems. J Exp Biol 202: 1855-1864.

Gelperin A. 2006. Olfactory computations and network oscillations. $J$ Neurosci 26: 1663-1668.

Gelperin A, Tank DW. 1990. Odor-modulated collective network oscillations of olfactory interneurons in a terrestrial mollusk. Nature 345: $437-440$.

Gelperin A, Rhines LD, Flores J, Tank DW. 1993. Coherent network oscillation by olfactory interneurons: Modulation by endogenous amines. J Neurophysiol 69: 1930-1939.

Gray CM, Konig P, Engel AK, Singer W. 1989. Oscillatory responses in cat visual cortex exhibit inter-columnar synchronization which reflects global stimulus properties. Nature 338: 334-337.

Hopfield JJ. 1995. Pattern recognition computation using action potential timing for stimulus representation. Nature 36: 33-36.

Hopfield JJ, Gelperin A. 1989. Differential conditioning to a compound stimulus and its components in the terrestrial mollusc Limax maximus. Behav Neurosci 103: 329-333.

Hughs JR, Mazurowski JA. 1962. Studies on the supracallosal mesial cortex of unanesthetized, conscious mammals. II Monkey. B. Responses 
from the olfactory bulb. Electroencephalogr Clin Neurophysiol 14: $635-645$.

Huxter J, Burgess N, O'Keefe J. 2003. Independent rate and temporal coding in hippocampal pyramidal cells. Nature 425: 828-832.

Inoue T, Murakami M, Watanabe S, Inokuma Y, Kirino Y. 2006. In vitro odor-aversion conditioning in a terrestrial mollusk. J Neurophysiol 95: 3898-3903.

Iwama A, Yamada A, Kimura T, Kono E, Sekiguchi T. 1997. A dual oscillator system in the procerebral lobe of Limax brain. Neurosci Res 21: 231.

Iwama A, Yabunaka A, Kono E, Kimura T, Yoshida S, Sekiguchi T. 1999. Properties of wave propagation in the oscillatory neural network in Limax marginatus. Zool Sci 16: 407-416.

Jensen O, Lisman JE. 2000. Position reconstruction from an ensemble of hippocampal place cells: Contribution of theta phase coding. J Neurophysiol 83: 2602-2609.

Kasai Y, Watanabe S, Kirino Y, Matsuo R. 2006. The procerebrum is necessary for odor-aversion learning in the terrestrial slug Limax valentianus. Learn Mem 13: 482-488.

Kawahara S, Toda S, Suzuki Y, Watanabe S, Kirino Y. 1997. Comparative study on neural oscillation in the procerebrum of the terrestrial slugs, Incilaria bilineata and Limax marginatus. J Exp Biol 200: 1851-1861.

Kimura T, Toda S, Sekiguchi T, Kirino Y. 1998a. Behavioral modulation induced by food odor aversive conditioning and its influence on the olfactory responses of an oscillatory brain network in the slug, Limax marginatus. Learn Mem 4: 365-375.

Kimura T, Suzuki H, Kono E, Sekiguchi T. 1998b. Mapping of interneurons that contribute to food aversive conditioning in the slug brain. Learn Mem 4: $376-388$.

Kimura T, Toda S, Sekiguchi T, Kawahara S, Kirino Y. 1998c. Optical recording analysis of olfactory response of the procerebral lobe in the slug brain. Learn Mem 4: 389-400.

Laurent G, Naraghi M. 1994. Odorant-induced oscillations in the mushroom bodies of the locust. J Neurosci 14: 2993-3004.

Li Z, Hopfield JJ. 1989. Modeling the olfactory bulb and its neural oscillatory processing. Biol Cybern 61: $379-392$.

Lubenov EV, Siapas AG. 2009. Hippocampal theta oscillations are traveling waves. Nature 459: 534-539.

Makinae H, Makino Y, Obara T, Yano M. 2008. Specific spatio-temporal activities in the cerebral ganglion of Incilarila fruhstorferi in response to superior tentacle nerve stimulation. Brain Res 1231: 47-62.

Markram H, Lübke J, Frotscher M, Sakmann B. 1997. Regulation of synaptic efficacy by coincidence of post- synaptic APs and EPSPs. Science 275: $213-215$.
O'Keefe J, Recce ML. 1993. Phase relationship between hippocampal place units and the EEG theta rhythm. Hippocampus 3: 317-330.

Ottoson D. 1959. Comparison of slow potentials evoked in the frog's nasal mucosa and olfactory bulb by natural stimulation. Acta Physiol Scand 47: $149-159$.

Ratte S, Chase R. 1997. Morphology of interneurons in the procerebrum of the snail Helix aspersa. J Comp Neurol 384: 359-372.

Sahley CL, Rudy JW, Gelperin A. 1981. An analysis of associative learning in a terrestrial mollusc. I. High-order conditioning, blocking and transient US pre-exposure effect. J Comp Physiol A 144: 1-8.

Sahley CL, Martin KA, Gelperin A. 1990. Analysis of associative learning in the terrestrial mollusc Limax maximus. II. Appetitive learning. J Comp Physiol A 167: 339-345.

Sekiguchi T, Yamada A, Suzuki H. 1997. Reactivation-dependent changes in memory states in the terrestrial slug Limax flavus. Learn Mem 4: $356-364$.

Shirahata T, Watanabe S, Kirino Y. 2004. Distribution of serotonin-like immunoreactive neurons in the slug Limax valentianus. Cell Tissue Res 315: $285-290$.

Skaggs WE, McNaughton BL, Wilson MA, Barnes CA. 1996. Theta phase precession in hippocampal neuronal populations and the compression of temporal sequences. Hippocampus 6: 149-172.

Stopfer M, Bhagavan S, Smith BH, Laurent G. 1997. Impaired odour discrimination on desynchronization of odour-encoding neural assemblies. Nature 390: 70-73.

Suzuki H, Sekiguchi T, Yamada A, Mizukami A. 1994. Sensory preconditioning in the terrestrial mollusc, Limax flavus. Zool Sci 11: $121-125$.

Teyke T, Gelperin A. 1999. Olfactory oscillations augment odor discrimination not odor identification by Limax CNS. Neuroreport 10: 1061-1068.

Watanabe S, Kirino Y, Gelperin A. 2008. Neural and molecular mechanisms of microcognition in Limax. Learn Mem 15: 633-642.

Wehr M, Laurent G. 1996. Odour encoding by temporal sequences of firing in oscillating neural assemblies. Nature 384: 162-166.

Yamada A, Kimura T, Kono E, Sekiguchi T. 1997. Simulation of the oscillatory potential propagation in the slug's brain with an array of nonlinear oscillators. In Complexity and diversity (ed. ER Nakamura), pp. 166-168. Springer-Verlag, Tokyo, Japan.

Zaitseva OV. 2000. Projectional connections and a hypothetical scheme of structural organization of procerebrums of terrestrial mollusks. J Evol Biochem Physiol 36: 604-620.

Received May 10, 2010; accepted in revised form June 24, 2010. 


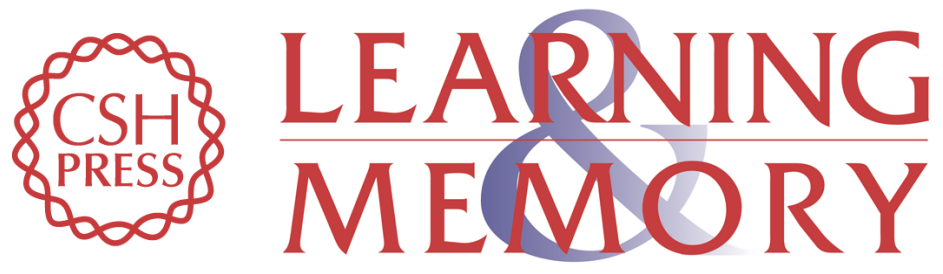

\section{Internal representation and memory formation of odor preference based on oscillatory activities in a terrestrial slug}

Tatsuhiko Sekiguchi, Hiroyuki Furudate and Tetsuya Kimura

Learn. Mem. 2010, 17:

Access the most recent version at doi:10.1101//m.1867110

References This article cites 48 articles, 12 of which can be accessed free at:

http://learnmem.cshlp.org/content/17/8/372.full.html\#ref-list-1

License

Email Alerting Receive free email alerts when new articles cite this article - sign up in the box at the Service top right corner of the article or click here. 